\title{
Research on emergency evacuation of subway station based on mobile capability
}

\author{
Jinxing Ying ${ }^{1, \mathrm{a})}$, Jin $\mathrm{Li}^{2, \mathrm{~b})}$ \\ ${ }^{1,2)}$ School of Management and E-Business, Contemporary Business and Trade Research Center, Zhejiang \\ Gongshang University, Hangzhou,310018, China \\ a)y1049119536@163.com \\ b) jinli@mail.zjgsu.edu.cn
}

\begin{abstract}
The rapid development of the subway contributes to public transportation, but at the same time the emergency evacuation of subway traffic puts forward higher requirements. At present, most of the studies on the emergency evacuation of the subway have neglected the differences in the mobility of different people in the subway stations, those studies are running out of time. In this paper, the evacuation population is divided into four categories to evacuate separately. Combining the theory of congestion, the relationship between different initial congestion degree and total evacuation time is obtained using dynamic programming method. The results show that when the population with different speed is evacuated separately, the optimal initial congestion degree exists and total evacuation time can reach minimum.
\end{abstract}

Keywords: emergency evacuation, subway, congestion degree

\section{INTRODUCTION}

Subway and other underground modes of transport extend the scope of urban transport and other public activities to the underground. That can effectively alleviate the public transport pressure of the city. In such a special environment, if the accident happened, such as fire and explosion, high temperature and smoke will cause extreme psychological panic, easily leading to group death. At present, the research on emergency evacuation of subway did not take account of the characteristics of the crowd. This paper explored and concluded the relationship between congestion degree and total evacuation time by dynamic planning, based on the rule that people who move at different speeds are evacuated separately. In the end of this paper, reliable suggestions are put forward from the result.

\section{THE CLASSIFICATION OF CROWD}

By analyzing the influencing factors of the moving speed, it is the most reasonable way to establish the classification according to the age structure considering the feasibility of actual operation. There is no significant difference among the data about age and moving speed provided by different articles, this paper refers to the data provided by Dou Lijie et al. (2013) to establish the crowd structure. The data is shown in Table 3:

Table 1. The statistic data of crowd structure in subway station [1].

\begin{tabular}{ccccc}
\hline Age Group & $<15$ & $15-30$ & $30-60$ & $>60$ \\
\hline Speed $(\mathrm{m} / \mathrm{s})$ & 1.096 & 1.242 & 1.184 & 1.151 \\
\hline Proportion(\%) & 5 & 51 & 38 & 6 \\
\hline
\end{tabular}

From table 1 , it can be concluded that the young adults having the normal ability account for a very large proportion in the subway, close to $90 \%$; children and the elderly with poor mobility only occupy a smaller proportion, close to $10 \%$. This proportion is consistent with the actual situation, because most of crowd in the subway are office workers. 


\section{ESTABLISHMENT OF SUBWAY MODEL}

Considering the geographical characteristics of the subway, emergency evacuation must be carried out from the bottom up, and stairs, escalators and corners are important bottlenecks of emergency evacuation [3]. The gate machine has an identical impact on the emergency evacuation, but it's confirmed that crowd can be evacuated within the specified time if the gates were working as usual [6]. The moving speed of crowd on the stairs is usually half of the moving speed on the ground [2], so we defined the length of stairs to double. Arranging evacuation management personnel to guide can alleviate well the pressure caused by disorder on the corners [4], so corners are not bottlenecks.

\section{Subway evacuation network}

The subway evacuation network is all accessible areas of the evacuation. In a subway evacuation network, the restriction facilities, such as corners, are defined as nodes, and the channel and the stairway between nodes are defined as links. On this basis, we construct the evacuation network $\mathrm{G}(\mathrm{N}, \mathrm{A}), \mathrm{N}$ is the node set, and $\mathrm{A}$ is the link set. Where $\mathrm{N}=(\mathrm{n} 1, \mathrm{n} 2, \ldots, \mathrm{ni})$, ni denotes the node $\mathrm{i}$.

The nodes do not have attributes, only as dividing points between the different channels. Due to the characteristics of subway station, most of nodes are narrow locations, such as staircase entrances and gates. Since this article assumes that when an accident occurred, there were managers who organized crowd at the corners to maintain order, the corners do not have limiting impact on evacuation, then it no longer has the ability to limit like a bottleneck. The attributes of the link include the length of path $l_{i j}$, the flow capacity $C_{i j}$ and the actual flow $V_{i j}$.

\section{ALGORITHM DESIGN}

According to the theory of congestion degree [5], when the congestion degree increases within a certain range, the moving speed of the crowd will be reduced accordingly. The relationship is as follows:

$$
v_{i j}=\left\{\begin{array}{l}
V_{\max } \exp \left(-0.5 * c_{i j}\right), c_{i j}>0.5 \\
V_{\max }, c_{i j} \leq 0.5
\end{array}\right\}
$$

$V_{\text {max }}$ is the maximum moving speed of the crowd;

$c_{i j}$ is the congestion degree of path $a_{i j}$;

$v_{i j}$ is the actual speed of crowd in the path $a_{i j}$;

The calculation formula for the traffic per second of each link is shown below:

$$
e_{i j}=\frac{V_{i j}}{v_{i j} / l_{i j}}
$$

$e_{i j}$ is the traffic per second of the path $a_{i j}$;

From the formula of congestion degree and speed, it can be concluded that with the increase of congestion degree, the speed of the crowd will slow down, so there may exist an optimal balance between congestion degree and crowd speed, so that the flow per second of crowd reaches the maximum value. The objective function is as follow:

$\mathrm{T}$ is the total evacuation time;

$$
\min T=\frac{Q}{f}
$$

$\mathrm{Q}$ is the number of crowd;

$f$ is the total flow per second of the exits;

\section{Steps of calculation:}

(1). Determine the priority of all feasible evacuation routes based on length;

(2). Get the flow per second $e_{i j}^{\max }$ of different age group with their maximum moving speed;

(3). Choose an initial congestion degree (C) and an age group which is classified with different speed, then we can know the initial flow $e_{i j}$ of first link by using the formula 1 and 2;

(4).The flow of the next link is depending on the previous link's flow and the current moving speed. If the flow $e_{i j}$ of the previous link exceeded the current flow $e_{j k}^{\max }$ which is the flow of the maximum speed of age group, 
reduction happened and we needed to recalculate the actual flow of the current link. If the flow $e_{i j}$ of the previous link did not exceed the $e_{j k}^{\max }$ of the current link, the actual flow $e_{j k}$ is equal to the previous flow;

(5). According to step 4, we can get the total flow of the last exits under the initial congestion degree (C);

(6). Based on the flow of each link and initial congestion degree, we can calculate the total time of the evacuation of the age group and the time of the last person spending which don't contain the waiting time;

(7). Replace the initial congestion degree and the age group, repeat step 2-6 until you determine the evacuation time and the time that the last person spent of different age groups at different initial congestion degree;

(8). Select the appropriate evacuation priority of age group, we can get the minimum total evacuation time;

\section{RESULTS AND ANALYSIS}

The potential of the evacuation route depends on the minimum capacity of the route. If various age groups were evacuated at the same time, it was bound to slow down the age group whose speed were faster because the moving speeds of various age groups are different, so that the total evacuation time must be longer. Therefore, it is the best choice that people with same speed move together.

When the initial congestion is not high, there may be only some routes used in the evacuation network. With the increase of congestion degree, the initial flow gradually increases and the rest routes of evacuation network will be used one by one. Only when the route with the highest priority was into the deceleration state, the congestion degree is over 0.5 , can the evacuees choose the route with the lower priority. When all of the routes were in the same deceleration state, the crowd will enter the shorter route.

Although there are various permutations, it is obvious that the shorter total time that crowd spend in waiting at the station, the shorter overall evacuation time. Therefore, the crowd was evacuated according to the priority of the speed, for example, the age group of higher moving speed was evacuated prior to the lower speed group.

This paper simulated the evacuation situation of the age group 15-30, as shown in Fig.1. When the moving speed of crowd is declining, the flow is increasing. The flow reaches the maximum, when the initial congestion degree increases to 2.0. It is probable to determine the best initial congestion degree according to moving speed and other related factors.

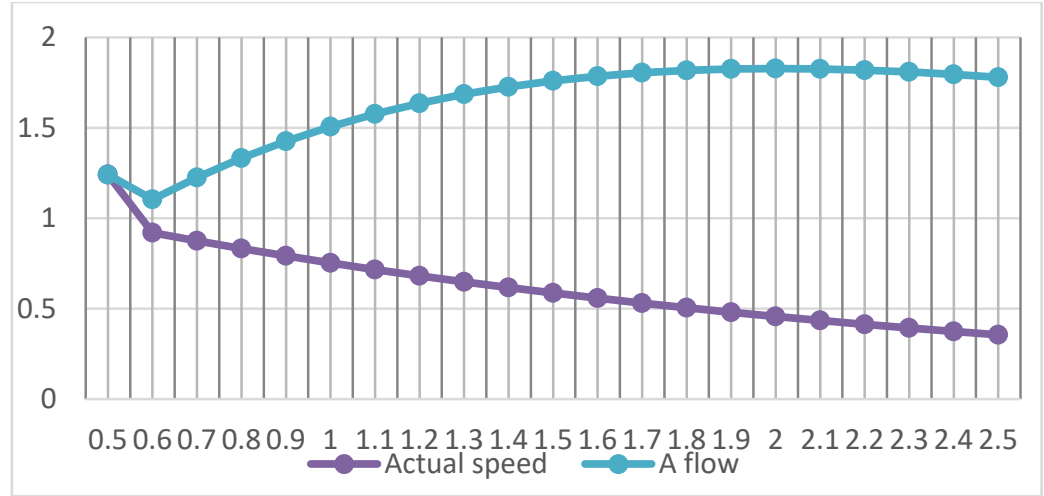

Figure 1.The relationship between actual speed and A flow under various congestion degrees.

In this paper, we use the method of dynamic programming to obtain the total evacuation time of the different congestion degree under the evacuation principle of the faster prior to the slower.

Fig.2 shows that when the congestion degree rises from 0.5, the evacuation time also increases, because the crowd density has reached the extent that will affect other people. When the initial congestion degree continues to rise, the total evacuation time is gradually reduced, which is consistent with the theory that increase of flow will reduce the total evacuation time. When the congestion degree reaches 1.7, the total evacuation time reaches the smallest state, indicating that the best congestion rate is 1.7 , and the total flow of the evacuation network is very close to the maximum output flow of the network. When initial congestion degree is more than 1.7, the rising of the initial congestion degree increases the total evacuation time. That suggests the potential of the evacuation network has been excavated. 


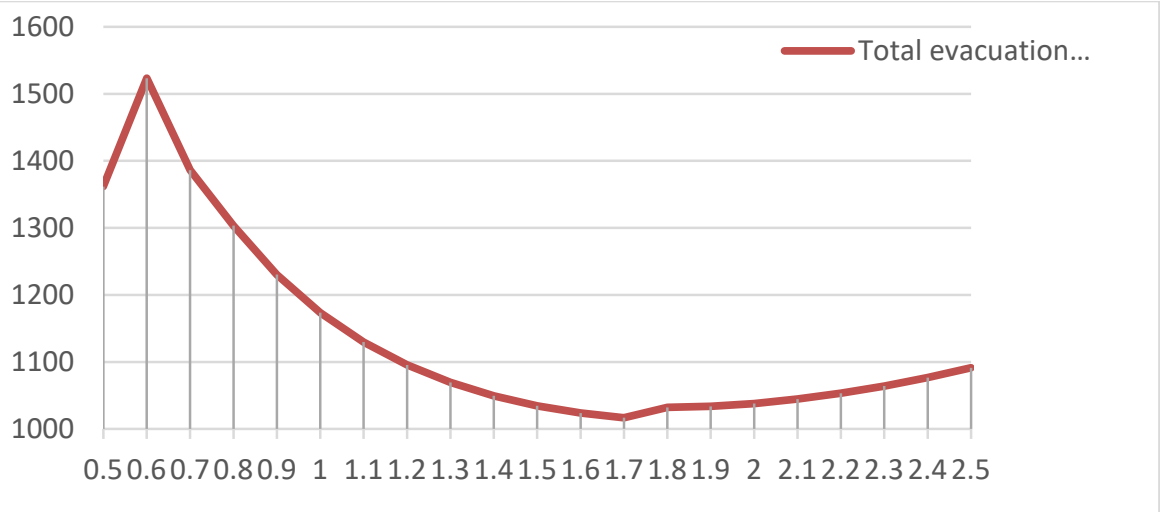

Figure 2. The relationship between initial congestion degree and total evacuation time.

\section{CONCLUSION}

The literatures on emergency evacuation suggest that there are many factors that affect the speed of crowd. After analyzing and considering the possibility of the actual operation, this paper chooses the main factors to carry on the research, and then establishes the classification with different speed. According to the theory of congestion degree, the relationship between congestion degree and total evacuation time is obtained by exhaustive method. The results show that there is an optimal congestion degree in the case of evacuation of different speed groups, and the total evacuation time is the minimum under the best initial congestion degree. Under the optimal congestion degree, the evacuation potential of evacuation network is fully excavated.

The optimal initial congestion degree of evacuation network is affected by factors such as moving speed and network capacity. In the actual evacuation plan, it is needed to collect a large amount of data to determine the optimal congestion degree. The initial congestion degree determined in this paper is relative to the whole group to be evacuated. In the actual evacuation, it would better determine different suitable congestion degrees for different crowd of different moving speed.

\section{ACKNOWLEDGEMENTS}

This work is partly supported by the Zhejiang Provincial Natural Science Foundation of China (Grant No. LY17G020005, LZ14G020001), Research Project of the Collaborative Innovation Center of Modern Trade Circulation System Construction of Zhejiang Gongshang University (Grant No. 15SMGK07D).

\section{REFERENCES}

1. Dou Lijie. Calculation and Analysis of Basic Parameters of Subway Personnel Evacuation [J]. Fire Science and Technology, 2013,32 (1): 19-21

2. Peter Thompson, Daniel Nilsson, Karen Boyce, Denise Mcgrath. Evacuation models are running out of time [J] .Fever Safety Journal, 2015, 78, 251-261

3. Wang Ruocheng.Study on bottleneck identification of subway personnel evacuation based on structural importance [J]. Journal of Safety Science and Technology, 2014,10 (12): 62-66

4. Ma Junchi, Li Jie, Zhou Xiyuan. Influence of road corner on evacuation [J]. Journal of Earthquake Prevention and Disaster Reduction Engineering, 2007,27 (4): 377-382

5. Li Qingquan, Li Qiuping, Fang Zhixiang. A Method for Optimization of Emergency Evacuation Path Based on Spatio-temporal Congestion [J]. Acta Sinica, 2011,40 (4): 517-523

6. Song Weiguo, Yu Yanfei, Chen Tao. The Influence of Export Conditions on Personnel Evacuation and Its Analysis [J] .2003,12 (2): 100-103 